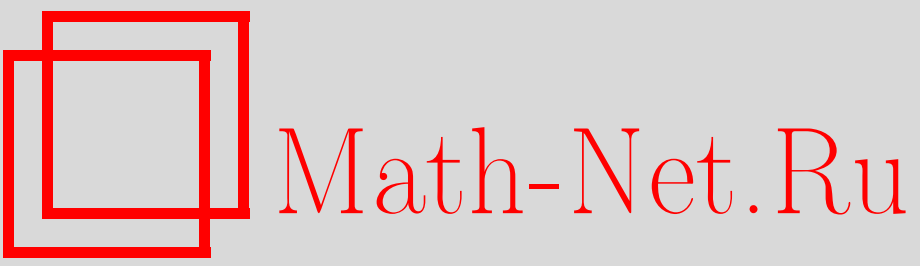

Л. Л. Буишвили, Л. Ж. Захаров, А. И. Тугуши, Квантовая диффузия в нерегулярных кристаллах, содержащих туннельные двухуровневые системы, ТМФ, 1996, том 106, номер 3, 489-497

DOI: https://doi.org/10.4213/tmf1131

Использование Общероссийского математического портала Math-Net.Ru подразумевает, что вы прочитали и согласны с пользовательским соглашением

http: //www . mathnet.ru/rus/agreement

Параметры загрузки:

IP : 52.6 .47 .48

26 апреля 2023 г., 12:29:19 
ТЕОРЕТИЧЕСКАЯ

И МАТЕМАТИЧЕСКАЯ

ФИЗИКА

Том 106, № 3

март, 1996

Л. Л. Буишвили, Л. Ж. Захаров, А.И. Тугуши ${ }^{1)}$

\section{КВАНТОВАЯ ДИФФУЗИЯ В НЕРЕГУЛЯРНЫХ КРИСТАЛЛАХ, СОДЕРЖАЩИХ ТУННЕЛЬНЫЕ ДВУХУРОВНЕВЫЕ СИСТЕМЫ}

Исследована задача квантовой диффузии примесных частиц, взаимодействующих с туннельными двухуровневыми системами (ДУС). Показано, что в температурной области $\omega_{0}<T \lesssim 1 \mathrm{~K}$ (где $\omega_{0}$ - среднее значение сбоя уровней энергии примесонов в соседних ямах) и при концентрации ДУС $N_{D} \gtrsim 10^{27}{ }_{\mathrm{M}}^{-3}$ коэффициент квантовой диффузии примесонов определяется взаимодействием примесонов с ДУС.

Посвящается светлой памяти Дмитрия Николаевича Зубарева

\section{1. ВВЕДЕНИЕ. ПОСТАНОВКА ЗАДАЧИ}

Исследованию явления квантовой диффузии было посвяшено большое число работ [1-4]. На характер квантовой диффузии примесных частиц существенное влияние оказывают различного рода статические и динамические флуктуации, имеющиеся в реальном кристалле. Статические флуктуации, вызванные нерегулярностью кристалла, а также наличием примесей, стремятся привести к локализации частиц, которая может преодолеваться слабым туннелированием. Что касается динамических флуктуаций, они в определенных случаях приводят к локализации частиц, в других же случаях способствуют делокализации. В нерегулярных кристаллах собственно квантовая диффузия примесонов может протекать лишь на фоне динамических (температурных) флуктуаций $[3,4]$.

Источником динамических флуктуаций обычно считают колебания решетки и учитывают взаимодействие примесных частиц с фононами. Эти вопросы детально изучались в работах [2-4]. Однако, как известно, при низких температурах $T \lesssim 1 \mathrm{~K}$ фононный вклад в кинетику процессов сильно подавляется.

Источником динамических флуктуаций могут быть также туннельные элементарные возбуждения, плотность состояний которых является величиной постоянной (или почти постоянной) [5]. Значение плотности состояний этих возбуждений может достигать значительных величин порядка $\bar{P} \sim 10^{46}$ Дж ${ }^{-1} \mathrm{M}^{-3}$, в то же время при низких температурах возбуждены в основном низкочастотные акустические фононы, плотность состояний которых значительно меньше, поэтому следует ожидать, что в образцах, содержащих туннельные двухуровневые системы (ДУС), динамика флуктуаций, влияющая на

\footnotetext{
${ }^{1)}$ Сотрудник Тбилисского государственного университета.
} 
кинетику квантовой диффузии, при низких температурах определяется связью примесонов с ДУС, а не с фононами.

В настоящей статье исследуется влияние туннельных ДУС на процессы квантовой диффузии.

В качестве примера кристаллов, содержаших туннельные ДУС, в которых наблюдаются процессы квантовой диффузии, можно привести водородосодержашие кристаллы ниобия и циркония.

Оператор взаимодействия примесных частиц с ДУС может быть получен по аналогии с тем, как это было проделано в работе [1], где выведено взаимодействие примесных частиц друг с другом. Действительно, ДУС является локальным образованием, имеющим два близколежаших состояния равновесия, переходы между которыми осушествляются благодаря туннельному эффекту [5], поэтому для получения оператора взаимодействия примесных частиц с ДУС достаточно представить расстояние между примесным атомом и ДУС в виде

$$
r_{S^{z}}=r+l S^{z}
$$

где $S^{z}$ - матрица Паули, $l$ - расстояние между соседними равновесными положениями атома в ДУС, $r$ - расстояние между центром ДУС и примесной частицей, которое учитьвает возможность того, что атом может находиться либо в одной, либо в соседней потенциальной яме.

Следовательно, оператор взаимодействия примет вид

$$
V_{0}=U(\mathbf{n}) \frac{d^{3}}{\left(r+l S^{z}\right)^{3}}
$$

где $d$ - межатомное расстояние, $\mathbf{n}=\frac{\mathbf{r}}{r}$ - единичный вектор, $U(\mathbf{n})$ - характерная энергия, зависяшая от взаимной ориентации примесного атома и ДУС. Учитывая то обстоятельство, что $r \gg l$, и проводя разложение по малому параметру $l / r$, получим

$$
V_{0}=U(\mathbf{n}) \frac{d^{3}}{r^{3}}-3 U(\mathbf{n}) \frac{l d^{3}}{r^{4}} S^{z}
$$

Первый член в (1) статический, вызываюший смещение уровней энергии примесных частиц в соседних ямах на величину $\Delta E \sim 3 U(\mathbf{n}) d^{4} / r^{4}$ тем большую, чем ближе расположена примесная частица к ДУС. Второй член в (2) описывает динамическое взаимодействие примесной частицы и ДУС. В диагональном по состояниям ДУС представлении (которое осушествляется посредством унитарного оператора $W \sim \exp \left(i \varphi S^{y}\right)$, где $\operatorname{tg} \varphi=\frac{\Delta_{0}}{\Delta}, \Delta$ - асимметрия ямы, $\Delta_{0}$ - туннельная энергия [5]) динамическая часть оператора взаимодействия примет вид

$$
V_{0}=-3 U(\mathbf{n}) \frac{l d^{3}}{r^{4}} \frac{\sqrt{\varepsilon^{2}-\Delta_{0}^{2}}}{\varepsilon} S^{z}-3 U(\mathbf{n}) \frac{l d^{3}}{r^{4}} S^{x}
$$

здесь $\varepsilon=\sqrt{\Delta^{2}+\Delta_{0}^{2}}-$ энергия расшепления ДУС. 
Запишем гамильтониан, описывающий задачу квантовой диффузии примесных частищ в узельном представлении [6]:

$$
\begin{aligned}
H & =H_{0}+V_{1}+V_{2}+V_{3}, \\
H_{0} & =\sum_{\mathbf{m}} E_{\mathbf{m}} \alpha_{\mathbf{m}}^{+} \alpha_{\mathbf{m}}+\sum_{k} \varepsilon_{k} S_{k}^{z}, \\
V_{1} & =\sum_{\mathbf{n} \neq \mathbf{m}} J_{\mathbf{n} \mathbf{m}} \alpha_{\mathbf{n}}^{+} \alpha_{\mathbf{m}}, \\
V_{2} & =\sum_{\mathbf{n} \mathbf{m} i} A_{\mathbf{n m} i} \alpha_{\mathbf{n}}^{+} \alpha_{\mathbf{m}} S_{i}^{z}+\sum_{\mathbf{n} \mathbf{m} i} B_{\mathbf{n m} i} \alpha_{\mathbf{n}}^{+} \alpha_{\mathbf{m}} S_{i}^{x}, \\
V_{3} & =\sum_{i j \alpha \beta} C_{i j}^{\alpha \beta} S_{i}^{\alpha} S_{j}^{\beta} .
\end{aligned}
$$

В операторе $H_{0}$ первый член описывает гамильтониан примесонов, $E_{\mathbf{m}}$ - энергия примесона, $\alpha_{\mathbf{m}}^{+}$и $\alpha_{\mathbf{m}}-$ операторы рождения и уничтожения примесной частицы в $\mathbf{m}-$ м узле, соответственно, второй член - гамильтониан системы ДУС, $V_{1}$ - туннельный оператор, $J_{\mathbf{n m}}$ - туннельная энергия. Оператор $V_{2}$ описывает взаимодействие примесонов с туннельными ДУС, причем те члены из $V_{2}$, в которых $\mathbf{n}=\mathbf{m}$, соответствуют процессам внутриузельных флуктуаций состояний примесонов, а члены с $\mathbf{n} \neq \mathbf{m}$ дают вклад в некогерентную диффузию. Как известно из работ [2-4], коэффициент некогерентной диффузии менше, чем коэффициент когерентной диффузии, поэтому в $V_{2}$ члены с $\mathbf{n} \neq \mathbf{m}$ рассматривать не будем. Оператор $V_{3}$ описывает взаимодействие между ДУС [7], которое при достаточно высокой концентрации ДУС определяет время корреляции псевдоспиновой корреляционной функции, которое в конечном счете формирует ширину уровней энергии примесонов:

$$
\begin{aligned}
& A_{\mathbf{n m} i}=-\int \psi^{*}(\mathbf{r}-\mathbf{n}) A(\mathbf{r}) \frac{\left(\varepsilon_{i}^{2}-\Delta_{0 i}^{2}\right)^{1 / 2}}{\varepsilon_{i}} \psi(\mathbf{r}-\mathbf{m}) d \mathbf{r}, \\
& B_{\mathbf{n m} i}=-\int \psi^{*}(\mathbf{r}-\mathbf{n}) A(\mathbf{r}) \frac{\Delta_{0 i}}{\varepsilon_{i}} \psi(\mathbf{r}-\mathbf{m}) d \mathbf{r}, \quad A(\mathbf{r})=U(\mathbf{n}) \frac{l d^{3}}{r^{4}},
\end{aligned}
$$

где $\psi(\mathbf{r}-\mathbf{n})$ - волновая функция примесона в $n$-м узле.

\section{2. ВЫВОД УРАВНЕНИЯ ДИФФУЗИИ}

Для получения уравнений диффузии и локального коэффициента диффузии можно воспользоваться методом неравновесного статистического оператора Д. Н. Зубарева [8], который для ансамбля диффундируюших частиц имеет вид

$$
\begin{aligned}
& \rho=Q^{-1} \exp \left\{-\beta\left(H_{0}-\int \mu(\mathbf{x}) n(\mathbf{x}) d \mathbf{x}+\int_{-\infty}^{0} e^{\xi t} d t \int \mu(\mathbf{x}) \dot{n}(\mathbf{x}, t) d \mathbf{x}\right)\right\}, \\
& Q=\operatorname{Sp} \exp \left\{-\beta\left(H_{0}-\int \mu(\mathbf{x}) n(\mathbf{x}) d \mathbf{x}+\int_{-\infty}^{0} e^{\xi t} d t \int \mu(\mathbf{x}) \dot{n}(\mathbf{x}, t) d \mathbf{x}\right)\right\}, \quad \xi \rightarrow 0,
\end{aligned}
$$

$n(\mathbf{x})$ - локальная плотность примесонов - определяется как

$$
n(\mathbf{x})=\sum_{\mathbf{g}} \alpha_{\mathbf{g}}^{+} \alpha_{\mathbf{g}} \delta(\mathbf{x}-\mathbf{g}) .
$$


Уравнение движения для оператора $n(\mathbf{x})$ имеет вид

$$
\dot{n}(\mathbf{x})=\frac{1}{i} \sum_{\mathbf{n} \mathbf{m}} J_{\mathbf{n} \mathbf{m}} \alpha_{\mathbf{n}}^{+} \alpha_{\mathbf{m}}\{\delta(\mathbf{x}-\mathbf{n})-\delta(\mathbf{x}-\mathbf{m})\},
$$

$\mu(\mathbf{x})$ - химический потенциал диффундируюших частищ, $\beta$ - обратная температура решетки, $\dot{n}(\mathbf{x}, t)$ - оператор $\dot{n}(\mathbf{x})$ в представлении Гайзенберга.

Для усреднения операторного уравнения движения для $n(\mathbf{x})$ разложим неравновесньй статистический оператора $\rho$ по степеням $\int_{-\infty}^{0} e^{\xi t} d t \int \mu(\mathbf{x}) \dot{n}(\mathbf{x}, t) d \mathbf{x}$ и, ограничиваясь линейным приближением по этой величине, получим

$$
\rho \approx\left\{1-\int_{0}^{1}\left(e^{-R \tau} F e^{R \tau}-\left\langle e^{-R \tau} F e^{R \tau}\right\rangle_{e}\right) d \tau\right\} \rho_{e}
$$

где

$$
R=\beta\left(H_{0}-\int \mu(\mathbf{x}) n(\mathbf{x}) d \mathbf{x}\right), \quad F=-\beta \int_{-\infty}^{0} e^{\xi t} d t \int \mu(\mathbf{x}) \dot{n}(\mathbf{x}, t) d \mathbf{x},
$$

$\rho_{e}=e^{-R} / \mathrm{Sp} e^{-R}-$ локально равновесное распределение. Учитьвая, что

$$
e^{-R \tau} \alpha_{\mathbf{n}}^{+} e^{R \tau}=\alpha_{\mathbf{n}}^{+} e^{-\beta\left(E_{\mathbf{n}}-\mu(\mathbf{n})\right) \tau}, \quad e^{-R \tau} \alpha_{\mathbf{n}} e^{R \tau}=\alpha_{\mathbf{n}} e^{\beta\left(E_{\mathbf{n}}-\mu(\mathbf{n})\right) \tau},
$$

для среднего значения $\dot{n}(\mathbf{x})$ получим

$$
\begin{aligned}
\langle\dot{n}(\mathbf{x})\rangle= & -\beta \sum_{\mathbf{n} \mathbf{m}} \sum_{\mathbf{i} \mathbf{j}} \int_{-\infty}^{0} e^{\xi t} d t\left\langle J_{\mathbf{n} \mathbf{m}} J_{\mathbf{i j}} \alpha_{\mathbf{n}}^{+} \alpha_{\mathbf{m}} \alpha_{\mathbf{i}}^{+}(t) \alpha_{\mathbf{j}}(t) \times\right. \\
& \left.\times\{\delta(\mathbf{x}-\mathbf{n})-\delta(\mathbf{x}-\mathbf{m})\}\left\{\int \mu(\mathbf{x})\{\delta(\mathbf{x}-\mathbf{i})-\delta(\mathbf{x}-\mathbf{j})\} d \mathbf{x}\right\}\right\rangle_{e} \times \\
& \times \int_{0}^{1} \exp \left(\beta\left(E_{\mathbf{j}}-E_{\mathbf{i}}+\mu(\mathbf{i})-\mu(\mathbf{j})\right)\right) \tau d \tau .
\end{aligned}
$$

После интегрирования по $d \mathbf{x}$ и $d \tau$ получим

$$
\begin{aligned}
\langle\dot{n}(\mathbf{x})\rangle= & -\sum_{\mathbf{n} \mathbf{m}} \sum_{\mathbf{i} \mathbf{j}} \int_{-\infty}^{0} e^{\xi t} d t\left\langle J_{\mathbf{n} \mathbf{m}} J_{\mathbf{i j}} \alpha_{\mathbf{n}}^{+} \alpha_{\mathbf{m}} \alpha_{\mathbf{i}}^{+}(t) \alpha_{\mathbf{j}}(t) \times\right. \\
& \times\{\delta(\mathbf{x}-\mathbf{n})-\delta(\mathbf{x}-\mathbf{m})\}\{\mu(\mathbf{i})-\mu(\mathbf{j})\}\rangle_{e} \times \\
& \times \frac{\exp \left\{\beta\left(E_{\mathbf{j}}-E_{\mathbf{i}}-\mu(\mathbf{j})+\mu(\mathbf{i})\right)\right\}-1}{E_{\mathbf{j}}-E_{\mathbf{i}}+\mu(\mathbf{i})-\mu(\mathbf{j})}
\end{aligned}
$$

$\langle\ldots\rangle_{e}$ означает усреднение по локально равновесному распределению. Принимая во внимание, что

$$
\left\langle\alpha_{\mathbf{n}}^{+} \alpha_{\mathbf{m}} \alpha_{\mathbf{i}}^{+}(t) \alpha_{\mathbf{j}}(t)\right\rangle_{e}=\left\langle\alpha_{\mathbf{n}}^{+} \alpha_{\mathbf{m}} \alpha_{\mathbf{m}}^{+}(t) \alpha_{\mathbf{n}}(t)\right\rangle_{e} \delta_{\mathbf{i m}} \delta_{\mathbf{j n}},
$$


из (2) будем иметь

$$
\begin{aligned}
\langle\dot{n}(\mathbf{x})\rangle= & -\int_{-\infty}^{0} e^{\xi t} d t \sum_{\mathbf{n m}} J_{\mathbf{n m}} J_{\mathbf{m} \mathbf{n}}\left\langle\alpha_{\mathbf{n}}^{+} \alpha_{\mathbf{m}} \alpha_{\mathbf{m}}^{+}(t) \alpha_{\mathbf{n}}(t)\right\rangle_{e} \times \\
& \times\{\delta(\mathbf{x}-\mathbf{n})-\delta(\mathbf{x}-\mathbf{m})\}\{\mu(\mathbf{m})-\mu(\mathbf{n})\} \frac{\exp \left(\beta \Omega_{\mathbf{n m}}\right)-1}{\Omega_{\mathbf{n m}}}
\end{aligned}
$$

где $\Omega_{\mathbf{n m}}=E_{\mathbf{n}}-E_{\mathbf{m}}-\mu(\mathbf{n})+\mu(\mathbf{m})$. В уравнении (3) можно формально разложить $\delta$-функцию в ряд Тейлора и ограничиться первым порядком разложения [8]:

$$
\delta(\mathbf{x}-\mathbf{n})-\delta(\mathbf{x}-\mathbf{m})=(\mathbf{n}-\mathbf{m}) \nabla_{\mathbf{m}} \delta(\mathbf{x}-\mathbf{m}) .
$$

Разложим также и локальные химические потенциалы диффундируюших частищ:

$$
\mu(\mathbf{n})-\mu(\mathbf{m})=(\mathbf{n}-\mathbf{m}) \nabla_{\mathbf{m}} \mu(\mathbf{m}) .
$$

Подставляя (4) и (5) в (3), получим

$$
\begin{aligned}
\langle\dot{n}(\mathbf{x})\rangle= & \int_{-\infty}^{0} e^{\xi t} d t \sum_{\mathbf{n} \mathbf{m}} J_{\mathbf{n m}} J_{\mathbf{m} \mathbf{n}}\left\langle\alpha_{\mathbf{n}}^{+} \alpha_{\mathbf{m}} \alpha_{\mathbf{m}}^{+}(t) \alpha_{\mathbf{n}}(t)\right\rangle_{e} \times \\
& \times(\mathbf{n}-\mathbf{m}) \nabla_{\mathbf{m}} \delta(\mathbf{x}-\mathbf{m})(\mathbf{n}-\mathbf{m}) \nabla_{\mathbf{m}} \mu(\mathbf{m}) \frac{\exp \left(\beta \Omega_{\mathbf{n} \mathbf{m}}\right)-1}{\Omega_{\mathbf{n} \mathbf{m}}}
\end{aligned}
$$

Введем примесонную корреляционную функцию $f(t)$ следующим образом:

$$
\left\langle\alpha_{\mathbf{n}}^{+} \alpha_{\mathbf{m}} \alpha_{\mathbf{m}}^{+}(t) \alpha_{\mathbf{n}}(t)\right\rangle_{e}=\left\langle\alpha_{\mathbf{n}}^{+} \alpha_{\mathbf{m}} \alpha_{\mathbf{m}}^{+} \alpha_{\mathbf{n}}\right\rangle_{e} e^{i\left(E_{\mathbf{m}}-E_{\mathbf{n}}\right) t} f(t)
$$

Тогда (6) примет вид

$$
\begin{aligned}
\langle\dot{n}(\mathbf{x})\rangle= & \sum_{\mathbf{n} \mathbf{m}} J_{\mathbf{n} \mathbf{m}} J_{\mathbf{m n}}\left\langle\alpha_{\mathbf{n}}^{+} \alpha_{\mathbf{m}} \alpha_{\mathbf{m}}^{+} \alpha_{\mathbf{n}}\right\rangle_{e} \times \\
& \times(\mathbf{n}-\mathbf{m}) \nabla_{\mathbf{m}} \delta(\mathbf{x}-\mathbf{m})(\mathbf{n}-\mathbf{m}) \nabla_{\mathbf{m}} \mu(\mathbf{m}) \frac{\exp \left(\beta \Omega_{\mathbf{n} \mathbf{m}}\right)-1}{\Omega_{\mathbf{n m}}} \times \\
& \times \int_{-\infty}^{0} e^{\xi t} f(t) \exp \left\{i\left(E_{\mathbf{m}}-E_{\mathbf{n}}\right) t\right\} d t .
\end{aligned}
$$

Ниже мы покажем, что $f(t)$ имеет лоренцев вид, поэтому

$$
\int_{-\infty}^{0} e^{\xi t} f(t) \exp \left\{i\left(E_{\mathbf{m}}-E_{\mathbf{n}}\right) t\right\} d t=\frac{1}{2} \varphi\left(\omega_{\mathbf{m} \mathbf{n}}\right),
$$

здесь $\varphi\left(\omega_{\mathbf{m n}}\right)$ - фурье-образ примесонной корреляционной функции, $\omega_{\mathbf{m} \mathbf{n}}=E_{\mathbf{m}}-E_{\mathbf{n}}$. Подставляя (8) в (7), будем иметь

$$
\begin{aligned}
\langle\dot{n}(\mathbf{x})\rangle= & \sum_{\mathbf{n} \mathbf{m}} J_{\mathbf{n} \mathbf{m}} J_{\mathbf{m} \mathbf{n}}\left\langle\alpha_{\mathbf{n}}^{+} \alpha_{\mathbf{m}} \alpha_{\mathbf{m}}^{+} \alpha_{\mathbf{n}}\right\rangle_{e} \frac{1}{2} \varphi\left(\omega_{\mathbf{m} \mathbf{n}}\right) \times \\
& \times(\mathbf{n}-\mathbf{m}) \nabla_{\mathbf{m}} \delta(\mathbf{x}-\mathbf{m})(\mathbf{n}-\mathbf{m}) \nabla_{\mathbf{m}} \mu(\mathbf{m}) \frac{\exp \left(\beta \Omega_{\mathbf{n m}}\right)-1}{\Omega_{\mathbf{n} \mathbf{m}}} .
\end{aligned}
$$


Ограничиваясь туннелированием между соседними узлами решетки (для чего будем полагать $\mathbf{n}=\mathbf{m}+\mathbf{d})$ и предполагая, что $\beta \Omega_{\mathbf{n m}}<1$, из (9) получим

$$
\begin{aligned}
\langle\dot{n}(\mathbf{x})\rangle= & \frac{1}{2} \beta \sum_{\mathbf{m}} J_{\mathbf{m}+\mathbf{d}, \mathbf{m}} J_{\mathbf{m}, \mathbf{m}+\mathbf{d}} \varphi\left(\omega_{\mathbf{m}+\mathbf{d}, \mathbf{m}}\right) \times \\
& \times \mathbf{d}\left\langle\alpha_{\mathbf{m}+\mathbf{d}}^{+} \alpha_{\mathbf{m}} \alpha_{\mathbf{m}}^{+} \alpha_{\mathbf{m}+\mathbf{d}}\right\rangle_{e} \cdot \nabla_{\mathbf{m}} \delta(\mathbf{x}-\mathbf{m}) \mathbf{d} \nabla_{\mathbf{m}} \mu(\mathbf{m}) .
\end{aligned}
$$

Проведя суммирование по $\mathbf{m}$, будем иметь

$$
\langle\dot{n}(\mathbf{x})\rangle=\frac{1}{2} \beta Z \mathbf{d} \nabla J_{\mathbf{x}+\mathbf{d}, \mathbf{x}} J_{\mathbf{x}, \mathbf{x}+\mathbf{d}}\left\langle\alpha_{\mathbf{x}+\mathbf{d}}^{+} \alpha_{\mathbf{x}} \alpha_{\mathbf{x}}^{+} \alpha_{\mathbf{x}+\mathbf{d}}\right\rangle_{e} \cdot \varphi\left(\omega_{\mathbf{x}+\mathbf{d}, \mathbf{x}}\right) \mathbf{d} \nabla \mu(\mathbf{x}),
$$

здесь $Z$ - число ближайших соседей.

Для химического потенциала используем выражение $\mu=T \ln C$ [9] ( $C$-относительная концентрация примесонов), поэтому для локального химического потенциала имеем выражение

$$
\mu(\mathbf{x})=T \ln \frac{\langle n(\mathbf{x})\rangle_{e}}{N} .
$$

С учетом (11) уравнение (10) примет вид

$$
\begin{aligned}
\langle\dot{n}(\mathbf{x})\rangle & =\frac{1}{2} Z \mathbf{d} \nabla J_{\mathbf{x}+\mathbf{d}, \mathbf{x}} J_{\mathbf{x}, \mathbf{x}+\mathbf{d}} \varphi\left(\omega_{\mathbf{x}+\mathbf{d}, \mathbf{x}}\right) \cdot \mathbf{d}\left\langle\alpha_{\mathbf{x}+\mathbf{d}}^{+} \alpha_{\mathbf{x}} \alpha_{\mathbf{x}}^{+} \alpha_{\mathbf{x}+\mathbf{d}}\right\rangle_{e} \frac{1}{\langle n(\mathbf{x})\rangle_{e}} \nabla\langle n(\mathbf{x})\rangle_{e}= \\
& =\sum_{i j} \frac{\partial}{\partial x_{i}} D^{i j} \frac{\partial\langle n(\mathbf{x})\rangle_{e}}{\partial x_{j}}
\end{aligned}
$$

где

$$
D^{i j}=\frac{1}{2} Z d^{i} d^{j} J_{\mathbf{x}+\mathbf{d}, \mathbf{x}} J_{\mathbf{x}, \mathbf{x}+\mathbf{d}} \frac{\left\langle\alpha_{\mathbf{x}+\mathbf{d}}^{+} \alpha_{\mathbf{x}} \alpha_{\mathbf{x}}^{+} \alpha_{\mathbf{x}+\mathbf{d}}\right\rangle_{e}}{\langle n(x)\rangle_{e}} \varphi\left(\omega_{\mathbf{x}+\mathbf{d}, \mathbf{x}}\right) .
$$

Введем среднее значение разности энергий примесонов в соседних узлах решетки $\omega_{0}$ и среднее значение энергии туннелирования между соседними узлами решетки $J_{0}$. Учитывая, что

$$
\left\langle\alpha_{\mathbf{x}+\mathbf{d}}^{+} \alpha_{\mathbf{x}} \alpha_{\mathbf{x}}^{+} \alpha_{\mathbf{x}+\mathbf{d}}\right\rangle_{e}=\langle n(\mathbf{x}+\mathbf{d})\rangle_{e}\left(1+\langle n(\mathbf{x})\rangle_{e}\right),
$$

проводя разложение по градиентам локальной концентрации примесонов и принимая во внимание, что $\langle n(\mathbf{x})\rangle_{e}<1$ и $\mathbf{d} \frac{\partial\langle n(\mathbf{x})\rangle_{e}}{\partial \mathbf{x}}<1$, выражение (13) перепишем в виде

$$
D^{i j}=\frac{1}{2} Z d^{i} d^{j} J_{0}^{2} \varphi\left(\omega_{0}\right)
$$

(Формулы написаны в системе единиц $\hbar=1, k_{5}=1$.) Здесь $d^{i}-$ компоненты вектора $\mathbf{d}$. Коэффициент диффузии $D^{i j}$ определяется туннельным гамильтонианом $V_{1}$ и той частью взаимодействия $V_{2}$, в которой взяты члены $\mathbf{c} \mathbf{n}=\mathbf{m}$. Пользуясь терминологией работ [2-4], можно назвать $D^{i j}$ коэффициентом когерентной диффузии.

\section{3. АНАЛИЗ КОРРЕЛЯЦИОННОЙ ФУНКЦИИ}

Время корреляции примесонной корреляционной функции, определяемое первым членом взаимодействия $V_{2} \mathbf{c} \mathbf{n}=\mathbf{m}$, можно вычислить методом, используемым в стохастической теории магнитного резонанса (см. [10]). 
Примесонная корреляционная функция определяется следующим образом:

$$
\left\langle\alpha_{\mathbf{n}}^{+} \alpha_{\mathbf{m}} \alpha_{\mathbf{m}}^{+}(t) \alpha_{\mathbf{n}}(t)\right\rangle_{e}=\left\langle\alpha_{\mathbf{n}}^{+} \alpha_{\mathbf{m}} \alpha_{\mathbf{m}}^{+} \alpha_{\mathbf{n}}\right\rangle_{e} f(t) \exp \left\{i\left(E_{\mathbf{m}}-E_{\mathbf{n}}\right) t\right\} .
$$

Временная зависимость операторов определяется как

$$
\alpha_{\mathbf{m}}^{+}(t) \alpha_{\mathbf{n}}(t)=\exp \left(i H^{\prime} t\right) \alpha_{\mathbf{m}}^{+} \alpha_{\mathbf{n}} \exp \left(-i H^{\prime} t\right)
$$

где

$$
H^{\prime}=\sum_{\mathbf{k}}\left(E_{\mathbf{k}}+\sum_{i} A_{\mathbf{k} \mathbf{k} i} S_{i}^{z}(t)\right) \alpha_{\mathbf{k}}^{+} \alpha_{\mathbf{k}}
$$

Мы учитываем тот факт, что из-за взаимодействия между ДУС, а также между ДУС и фононами $S_{i}^{z}$ зависит от времени.

Уравнение движения для $\alpha_{\mathbf{m}}^{+}(t) \alpha_{\mathbf{n}}(t)$ имеет вид

$$
\frac{d}{d t} \alpha_{\mathbf{m}}^{+}(t) \alpha_{\mathbf{n}}(t)=\alpha_{\mathbf{m}}^{+}(t) \alpha_{\mathbf{n}}(t)\left\{i\left(E_{\mathbf{m}}-E_{\mathbf{n}}\right)+i \sum_{k}\left(A_{\mathbf{m m} k}-A_{\mathbf{n n} k}\right) S_{k}^{z}(t)\right\} .
$$

Решая это уравнение, для $f(t)$ получим

$$
f(t)=\left\langle\exp \left\{i \int_{0}^{t}\left(A_{\mathbf{m m} k}-A_{\mathbf{n n} k}\right) S_{k}^{z}\left(t^{\prime}\right) d t^{\prime}\right\}\right\rangle_{e}
$$

Заменим $S_{k}^{z}(t)$ на соответствующую флуктуационную часть и учтем, что $\left\langle\delta S_{k}^{z}(t)\right\rangle_{e}=0$. Определим величину

$$
\sigma^{2}=\left\langle\left(\sum_{k}\left(A_{\mathbf{m m} k}-A_{\mathbf{n n} k}\right) \delta S_{k}^{z}\right)^{2}\right\rangle_{e}=\frac{\bar{P} V}{2}\left(A_{\mathbf{m m}}-A_{\mathbf{n n}}\right)^{2} T \ln \frac{\varepsilon_{\max }}{l \Delta_{0}} .
$$

Поскольку мы ограничиваемся туннелированием между соседними узлами, то для $\sigma^{2}$ получим

$$
\sigma^{2}=\frac{\bar{P} V}{2}\left(A_{\mathbf{m m}}-A_{\mathbf{m}+\mathbf{d}, \mathbf{m}+\mathbf{d}}\right)^{2} T \ln \frac{\varepsilon_{\max }}{l \Delta_{0}}
$$

здесь $V$ - объем, приходяшийся на одну ДУС, $T$ - температура решетки, $\varepsilon_{\max }-$ максимальная энергия ДУС, $\Delta_{0}$ - минимальная энергия туннелирования между двумя равновесными положениями в ДУС.

В условиях $\sigma^{-1}>\tau_{D}$ [10] (где $\tau_{D}$ - время корреляции корреляционной функции $\left.\left\langle\delta S_{k}^{z} \delta S_{k}^{z}(t)\right\rangle_{e}\right) f(t)$ имеет лоренцев вид, а время корреляции примесонной корреляционной функции выражается по формуле

$$
\frac{1}{\tau_{\mathbf{m}, \mathbf{m}+\mathbf{d}}^{(1)}}=\sigma^{2} \tau_{D}
$$

$\tau_{D}$ определяется взаимодействием ДУС друг с другом и имеет вид [11]

$$
\frac{1}{\tau_{D}}=\frac{\bar{P} N_{D} \gamma^{4} B^{-1 / 2}}{1,8 \hbar\left(64 \pi \rho v^{2}\right)^{2}}
$$


где $\rho$ - плотность образца, $v$ - средняя скорость звука, $N_{D}$ - концентрация ДУС, $B$ - численный коэффициент порядка $10^{-2}$. Соотношение $\tau_{D}<\sigma^{-1}$ при температурах $T \sim 1 \mathrm{~K}$ выполняется в том случае, когда концентрация ДУС удовлетворяет неравенству $N_{D}>10^{24} \mathrm{M}^{-3}$ (считаем, что $U \sim 0,1$ эВ [12]).

С понижением температуры все большую роль в определении времени корреляции корреляционной функции играет второй член из $V_{2} \mathbf{c} \mathbf{n}=\mathbf{m} . \mathrm{C}$ помощью теории возмущений легко получить, что время корреляции, даваемое вторым членом из $V_{2}$, выражается по формуле

$$
\frac{1}{\tau_{\mathbf{m}, \mathbf{m}+\mathbf{d}}^{(2)}}=\frac{1}{4} \bar{P} V \pi\left(A_{\mathbf{m m}}-A_{\mathbf{m}+\mathbf{d}, \mathbf{m}+\mathbf{d}}\right)^{2} .
$$

Сравнение $\tau_{\mathbf{m}, \mathbf{m}+\mathbf{d}}^{(1)} \mathrm{c} \tau_{\mathbf{m}, \mathbf{m}+\mathbf{d}}^{(2)}$ показывает, что при концентрации ДУС $N_{D} \sim 10^{27} \mathrm{M}^{-3}$ и при $T>10^{-2} \mathrm{~K}$ имеем $\tau_{\mathbf{m}, \mathbf{m}+\mathbf{d}}^{(1)}<\tau_{\mathbf{m}, \mathbf{m}+\mathbf{d}}^{(2)}$. Противоположный предельный случай находится вне области применимости выведенного уравнения диффузии $\left(T>\omega_{0} \sim 0,1 \mathrm{~K}\right)$.

В вышеприведенных формулах мы не учитывали фононного вклада в ширину уровней, полагая его малым. При наличии фононов очевидно, что время корреляции $\tau_{\mathbf{m}, \mathbf{m}+\mathbf{d}}^{(1)}$ следует заменить на $\tau_{\text {eff: }}$

$$
\frac{1}{\tau_{\mathrm{eff}}}=\frac{1}{\tau_{\mathbf{m}, \mathbf{m}+\mathbf{d}}^{(1)}}+\frac{1}{\tau_{\mathrm{ph}}}
$$

$\tau_{\mathrm{ph}}$ определяется взаимодействием примесонов с фононами. Величина $\tau_{\mathrm{ph}}$ была рассчитана в работе [3], где исследовался вклад одно- и двухфононных процессов. Сравнение $\tau_{\mathbf{m}, \mathbf{m}+\mathbf{d}}^{(1)}$ с вкладом однофононных процессов показывает, что при $N_{D} \gtrsim 10^{27} \mathrm{M}^{-3}$ и $U \sim 0,1 э$ В связь примесонов с ДУС является определяющей.

\section{4. ЗАКЛЮЧЕНИЕ}

Таким образом, окончательно для коэффициента диффузии получим выражение

$$
D^{i j}=\frac{1}{2} Z d^{i} d^{j} J_{0}^{2} \frac{1}{\omega_{0}^{2} \tau_{\mathbf{m}, \mathbf{m}+\mathbf{d}}^{(1)}}=\frac{1}{2} Z d^{i} d^{j} J_{0}^{2} \frac{\sigma^{2} \tau_{D}}{\omega_{0}}
$$

здесь учтено, что $\omega_{0} \tau_{\mathbf{m}, \mathbf{m}+\mathbf{d}}^{(1)}>1$.

Заметим, что если в формулах (14) и (15) время корреляции $\tau_{\mathbf{m}, \mathbf{m}+\mathbf{d}}^{(1)}$ заменить на $\tau_{\mathrm{ph}}$, то полученные результаты будут совпадать с результатами работ [2-4].

В заключение отметим, что взаимодействие примесонов с ДУС не меняет линейного характера температурной зависимости коэффициента квантовой диффузии при низких температурах. Наряду с этим переход к сильной температурной зависимости $\left(D \sim T^{9}\right)$, обусловленной вкладом двухфононных процессов, осушествляется при более высоких температурах $(T \sim 10 \mathrm{~K})$, чем это происходит в отсутствие ДУС.

Настояшая работа была поддержана Международным научным фондом (грант № МХ $\mathrm{K} 000)$.

\section{Список литературы}

[1] Андреев А. Ф. // УФН. 1976. Т. 118. Вып. 2. С. 251-271. 
[2] Каган Ю., Максимов Л. А. // ЖЭТФ. 1973. Т. 65. С. 622-632.

[3] Каган Ю., Максимов Л. А. // ЖЭТФ. 1983. Т. 84. Вып. 2. С. 792.

[4] Каган Ю., Максимов Л. А. // ЖЭТФ. 1980. Т. 79. С. 1363.

[5] Бләк Джс. // Металлические стекла / Eds. Г. Гюнтеродт, Г. Бек. М.: Мир, 1983.

[6] Давьдов А.С. Теория твердого тела. М.: Наука, 1976.

[7] Смоляков Б. П., Хаймович Е. П. // УФН. 1982. Т. 136. Вып. 2. С. 317.

[8] Зубарев Д. Н. Неравновесная статистическая термодинамика. М.: Наука, 1971.

[9] Ландау Л. Д., Лифиич Е. М. Статистическая физика. М.: Наука, 1975.

[10] Kубо P. // Термодинамика необратимых процессов / Ред. Д. Н. Зубарев. М.: ИЛ, 1962.

[11] Szeftel J., Alloul H. // J. Non-Cryst. Solids. 1978. V. 29. P. 253-281.

[12] Qi Zh., Volkl J., Lasser R., Wenzl H. // J. Phys. F. 1983. V. 13. P. 2053-2062.

Институт физики

АН Грузии

Поступила в редакцию 24.IV.1995 г.

L. L. Buishvili, L. J. Zakharov, A. I. Tugushi

QUANTUM DIFFUSION IN IRREGULAR CRYSTALS HAVING TUNNEL TWO-LEVEL SYSTEMS

The quantum diffusion of admixture particles interacting with tunnel two-level systems is studied. It is shown that in the temperature region $\omega_{0}<T \lesssim 1 \mathrm{~K}$, where $\omega_{0}$ is an average value of the energy disturbance of admixture particles in the neighbouring pits, and under the concentration $N_{D} \gtrsim 10^{27} \mathrm{~m}^{-3}$ of two-level systems the quantum diffusion coefficient of admixture particles is determined by their interaction with two-level systems. 